\title{
Protriptyline treatment of sleep hypoxaemia in Duchenne muscular dystrophy
}

\author{
P E M SMITH, R H T EDWARDS, P M A CALVERLEY \\ From the Muscle Research Centre, University Department of Medicine and Regional Thoracic Unit, Fazakerley \\ Hospital, Liverpool
}

ABSTRACT Protriptyline $20 \mathrm{mg}$ daily reduced the total time spent in rapid eye movement sleep in an open study in four subjects with Duchenne muscular dystrophy. Sleep related hypoxaemia and유 episodes of desaturation were reduced. Anticholinergic side effects were prominent, however, in theseo patients, precluding its use for regular treatment.

\section{Introduction}

Patients with very weak inspiratory muscles are vulnerable to recurrent hypoxaemia during rapid eye movement (REM) sleep, ${ }^{1}$ possibly as a result of the reduction in intercostal and accessory muscle activity that normally accompanies this sleep stage. Pharmacological suppression of REM sleep is a possible therapeutic approach to REM related hypoxaemia. Protriptyline, a non-sedating tricyclic antidepressant, has been used successfully for the treatment of classical sleep apnoea syndromes. ${ }^{2}$ We describe a short term open study of protriptyline in five patients with advanced Duchenne muscular dystrophy who had REM related hypoxaemia but described no abnormal sleep symptoms or hypersomnolence.

\section{Methods}

Five patients aged 18-21 (mean 18.8) years with Duchenne muscular dystrophy, but with minimal scoliosis and no abnormal sleep symptoms, entered the study. REM related sleep hypoxaemia ( $>10 \%$ oxygen desaturation) had been documented in all the subjects. Vital capacity (VC), maximum inspiratory (MIP) and expiratory (MEP) static mouth pressures, and arterial blood gas tensions were measured by standard methods. Standard polysomnography methods, with an acclimatisation night, were used. ${ }^{\prime}$ The patients were positioned and turned during the study as necessary by a parent, who slept nearby. No

Address for reprints requests: Dr P M A Calverley, Mersey Regional Thoracic Unit, Fazakerley Hospital, Liverpool L19 7AL.

Accepted 22 September 1989 particular sleeping posture was imposed. After the $\stackrel{\mathbb{D}}{-}$ baseline study the subjects were prescribed protrip-tyline $20 \mathrm{mg}$ daily for 14 days, after which they were studied again with a further acclimatisation night. The $\overrightarrow{0}$ drug was then discontinued. A symptom question- -0 naire was completed after the study.

Episodes of desaturation were classified as falls of to least $5 \%$ or $10 \%$ from the preceding stable (usuatiy non-REM) value. Definitions of hypopnoea, apnoêa, arousals, and awakenings and the criteria for sleep staging and heart rate estimation were as described previously. ${ }^{1}$ Mean (SEM) values were compared by을 Student's $t$ test and correlation between mean valuesfor the different variables for each subject by regression analysis.

\section{Results}

Four patients completed the study. One 15 year old 3 . patient (VC 1.0 litre, MIP $30 \mathrm{~cm} \mathrm{H} \mathrm{H}_{2} \mathrm{O}$, MEP $20 \mathrm{~cm}$ $\mathrm{H}_{2} \mathrm{O}$ ) withdrew before the second study with intolera- 3 ble constipation and a thrombosed haemorrhoid aftero seven days. The four remaining patients also reported anticholinergic symptoms - dry mouth (three), con-o stipation (three), micturition difficulty (two) blurred vision (one).

The mean (range) pulmonary function values of then four patients included: VC 1.0 (0.7-1.3) litre, MIP 31.3N (25-40) $\mathrm{cm} \mathrm{H}_{2} \mathrm{O}$ and MEP 26.3 (15-40) $\mathrm{cm} \mathrm{H}_{2} \mathrm{O}$; when they were breathing air the arterial carbon dioxide tension $\left(\mathrm{PaCO}_{2}\right)$ was $5.5(4.8-6.05) \mathrm{kPa}$ and oxygen tension $\left(\mathrm{PaO}_{2}\right) 14.2(12.5-17.9) \mathrm{kPa}$. The table shows? mean sleep times, the proportion and latency of REM $_{T}$ sleep, oxygen saturation, characteristics of hypopnoea and apnoea, and frequency of arousals and awaken-两 
Mean (range] (SEM) values from baseline and protriptyline sleep studies of the four patients: total sleep time (TST) and percentage of TST in stage REM (\%SR); REM onset latency excluding periods of wakefulness (ROL excluding W); oxygen saturation $\left(\mathrm{SaO}_{2}\right)$ awake and in non-REM sleep and desaturation frequencies per hour slept; episodes of hypopnoea or apnoea; arousal and awakening frequencies

\begin{tabular}{lcc}
\hline & Baseline & Protriptyline \\
\hline TST (min) & $388[352-439]$ & $412[349-448]$ \\
$\%$ SR $(\%)$ & $19 \cdot 7[14-29]$ & $5 \cdot 5[0-12]$ \\
ROL (excluding W) (min) & $106[73-168]$ & $>277[198->349]$ \\
SaO $(\%)$ & $96 \cdot 2[95 \cdot 5-97 \cdot 0]$ & $96 \cdot 1[95 \cdot 0-98 \cdot 0]$ \\
Awake & $94 \cdot 5[94 \cdot 5-96 \cdot 0]$ & $95 \cdot 5[94 \cdot 0-98 \cdot 0]$ \\
Non-REM sleep & $5 \cdot 7[1 \cdot 4-16 \cdot 1]$ & $1 \cdot 2[0-2 \cdot 8]$ \\
No of desaturations/h & $3 \cdot 2[0 \cdot 3-10 \cdot 7]$ & $0 \cdot 4[0-1 \cdot 2]$ \\
$>5 \%$ & $81 \cdot 0(18 \cdot 9)$ & $28 \cdot 3(12 \cdot 3)^{*}$ \\
$>10 \%$ & $27 \cdot 1(2 \cdot 5)$ & $18 \cdot 9(2 \cdot 2)^{*}$ \\
Hypopnoea or apnoea & $12 \cdot 6(2 \cdot 9)$ & $4 \cdot 0(1 \cdot 8)^{*}$ \\
Total No of episodes & $12 \cdot 4[6 \cdot 2-20 \cdot 8]$ & $9 \cdot 3[5 \cdot 6-15 \cdot 6]$ \\
Duration (s) & $0 \cdot 9[0 \cdot 3-1 \cdot 1]$ & $0 \cdot 9[0 \cdot 5-1 \cdot 4]$ \\
Frequency/h & &
\end{tabular}

${ }^{*} p<0.05$ for significance of differences between means

ings for the four patients who were studied twice. ged in one, the overall mean reduction being $67 \cdot 3 \%$ Mean sleep efficiency (total sleep time (TST) as a (14.8-100\%). The latency of onset (excluding awake proportion of the study duration) was similar on the baseline $(76 \cdot 4 \%)$ and protriptyline $(79 \cdot 9 \%)$ nights. The proportion of TST by REM sleep (\%SR) was greatly reduced in three subjects but almost unchantime) of REM sleep was also greatly increased in three (no REM sleep being recorded in one of these) but little changed in one.

The figure illustrates typical suppression of REM
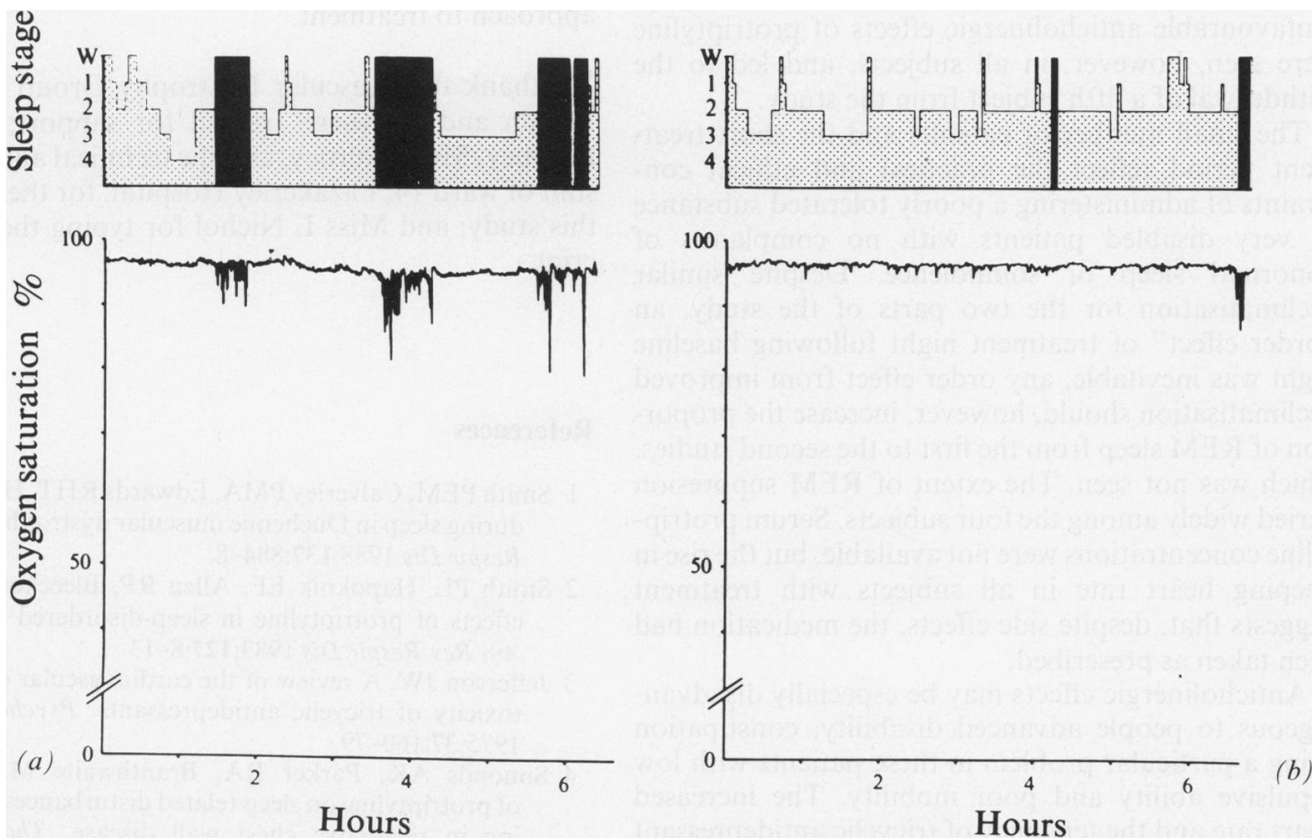

Data from patient 2 (part $b$ abbreviated for clarity) showing REM sleep suppression during protriptyline treatment with associated improvement in REM related hypoxaemia. Shaded areas: $W$-awake; solid barsREM sleep; 1-4 represents stages 1-4 of non-REM sleep. 
sleep and improvement in REM associated oxygen desaturation with protripyline. One patient, who had REM related desaturation of over $10 \%$ in the baseline study, showed no REM sleep and no oxygen desaturation with protriptyline.

The proportion of total sleep occupied by hypopnoea or apnoea was reduced during protriptyline treatment in all patients by $45-95 \%$, from a mean (SEM) $\%$ of $10.1(3.1)$ to $2.6(1 \cdot 1)(\mathrm{p}<0.05)$. The extent of REM suppression was correlated with the percentage reduction in $5 \%$ desaturation frequency $(\mathrm{r}=0.956(\mathrm{p}<0.05))$ and with the reduction in hypopnoea or apnoea with protriptyline $(r=0.997$, $\mathrm{p}<0.01)$ ). The frequency and duration of non-REM events also fell in all subjects having the treatment (baseline 3.98 events/hour REM none sleep (mean duration 23.4 seconds); protriptyline 1.57 events/hour non-REM (duration 15.6 s)).

The mean heart rate in stable non-REM sleep increased in all subjects during protriptyline treatment by $21-47 \%$ (mean $32.6 \%$ ), from a mean of 75.8 to 99.6 beats/min. Similar changes were seen in REM sleep.

\section{Discussion}

Protriptyline was effective in suppressing REM sleep, thereby reducing oxygen desaturation and disordered breathing during sleep in three out of four subjects with advanced Duchenne muscular dystrophy. Unfavourable anticholinergic effects of protriptyline were seen, however, in all subjects, and led to the withdrawal of a fifth subject from the study.

The small number of patients and the short treatment period reflect the practical and ethical constraints of administering a poorly tolerated substance to very disabled patients with no complaints of abnormal sleep or somnolence. Despite similar acclimatisation for the two parts of the study, an "order effect" of treatment night following baseline night was inevitable; any order effect from improved acclimatisation should, however, increase the proportion of REM sleep from the first to the second studies, which was not seen. The extent of REM suppresion varied widely among the four subjects. Serum protriptyline concentrations were not available, but the rise in sleeping heart rate in all subjects with treatment suggests that, despite side effects, the medication had been taken as prescribed.

Anticholinergic effects may be especially disadvantageous to people advanced disability, constipation being a particular problem in these patients with low expulsive ability and poor mobility. The increased heart rate and the tendency of tricyclic antidepressant drugs to precipitate arrhythmias ${ }^{3}$ may present a specific hazard to patients with Duchenne muscular dystrophy as they already have a high prevalence of electrocardiographic conduction abnormalities and cardiomyopathy.

The main potential benefit of protriptyline too patients with sleep disordered breathing relates to its ability to suppress REM sleep, ${ }^{24}$ though suppression $\overline{\bar{N}}$ varies between individuals. Data from animals suggest $\Phi$ that enhancement of efferent activity to the upper airway muscles by protriptyline may contribute to the ${ }^{\text {क }}$ relief of obstructive sleep apnoea, ${ }^{5}$ perhaps explaining. $\vec{\circ}$ the apparent influence of protriptyline in non-REM $\overrightarrow{-}$ sleep, ${ }^{2}$ as observed in our subjects. Protriptyline has ${ }^{\omega}$ previously been used effectively in the treatment of patients with symptomatic obstructive sleep apnoea ${ }^{26} \times$ and in patients with cardiorespiratory failure from + scoliosis or restrictive lung disease or both. ${ }^{4}$ Its $\vec{N}$ reported use in patients with primary neuromuscular $\vec{B}$ disease has been limited to isolated cases of myotonic dystrophy, ${ }^{7}$ postpoliomyelitis syndrome, ${ }^{89}$ ando nemaline myopathy, ${ }^{10}$ with few details of polysomnographic studies. Its use in Duchenne muscular dystrophy has not previously been reported.

Although suppression of REM sleep improves both $\stackrel{\mathbb{}}{\stackrel{\mathbb{T}}{3}}$ sleep disorderd breathing and the associated hypoxaemia during sleep in Duchenne muscular dystrophy, protriptyline appears to be inappropriate for this $\oplus_{\infty}$ purpose because of adverse anticholinergic effects;.$\oplus$ occasionally REM sleep may still occur normaly despite such side effects. Suppression of REM sleep a non-tricyclic agent may provide an alternat approach to treatment.

We thank the Muscular Dystrophy Group of Great Britain and Northern Ireland for support; Miss $\mathrm{H}$ 을 Downey, Mr H Gurney, and the technical and nursing staff of ward 14, Fazakerley Hospital, for their help in this study; and Miss L Nichol for typing the manuscript.

\section{References}

1 Smith PEM, Calverley PMA, Edwards RHT. Hypoxemia 윽 during sleep in Duchenne muscular dystrophy. Am RevD Respir Dis 1988;137:884-8.

2 Smith PL, Hapoknik EF, Allen RP, Bleecker ER. The effects of protriptyline in sleep-disordered breathing. Am Rev Respir Dis 1983;127:8-13.

3 Jefferson JW. A review of the cardiovascular effects andN toxicity of tricyclic antidepressants. Psychosom Med 1975;37:160-79.

4 Simonds AK, Parker RA, Branthwaite MA. Effect of protriptyline on sleep related disturbances of breath- $\Phi$ ing in restrictive chest wall disease. Thorax $1986 ;$ 41:586-90.

5 Bonora M, St John WM, Bledsoe TA. Differentia $\bar{p}$ elevation by protriptyline and depression by diazeparf 
of upper airway respiratory motor activity. Am Rev Respir Dis 1985;131:41-5.

6 Conway WA, Zorick F, Piccione P, Roth T. Protriptyline in the treatment of sleep apnoea. Thorax 1982;37: 49-53.

7 Clarke RW, Schmidt HS, Schaal SF, Boudoulas H, Schuller DE. Sleep apnea: treatment with protriptyline. Neurology 1979;29:1287-92.

8 Fischer DA. Poliomyelitis: late pulmonary complications and management. In: Halstead LS, Wiechers DO, eds.
Late effects of poliomyelitis. Miami: Symposia Foundation, 1985:185-92.

9 Olson LG, Hensley MJ, Sullivan CE, Saunders NA. Sleep, breathing and lung disease. In: Sullivan CE, Saunders NA, eds. Sleep and breathing. New York: Dekker, 1984:517-58.

10 Harati Y, Niakan E, Bloom K, Casar G. Adult onset of nemaline myopathy presenting as diaphragmatic paralysis. J Neurol Neurosurg Psychiatry 1987;50: 109-10.

\section{Book notices}

Case Presentations in Respiratory Medicine. John A Elliott. (Pp 211; £11.95.) Guildford: Butterworth, 1989. ISBN 0407 008101.

This excellent little book is the latest (and welcome) addition to Butterworth's series of case presentations. John Elliott presents 50 cases covering a broad spectrum of respiratory diseases. The presentations are well written, succinct, and for the most part unambiguous. Two thirds of the book is devoted to discussion of the cases-and herein lies its strength. When appropriate the differential diagnosis is discussed, followed by a more detailed consideration of the case in question, including management. The sections on pulmonary vasculitis, collagen vascular disease, sarcoidosis, and airflow obstruction are particularly good. Most discussions are accompanied by one or two key references, though these occasionally refer to textbooks that will not be accessible to all. The text is indexed, in keeping with the fact that this book is more than a series of questions and answers. Inevitably a book of this size cannot cover all aspects of respiratory medicine, but it does provide postgraduate doctors with a series of well chosen diagnostic challenges, which should help them with both MRCP examinations and day to day medical practice. I would have liked more discussion on the respiratory complications of immunodeficiency, which are mentioned only briefly-and, astoundingly, the highly topical subject of AIDS is not mentioned at all. Likewise, cryptogenic organising pneumonitis, also topical, is not considered among the differential diagnoses of diffuse, bilateral radiographic shadows. Those who read this book will find that they have painlessly digested what amounts to a small textbook of respiratory medicine. I can recommend the book for postgraduate doctors taking MRCP examinations, and in particular for more junior doctors working in respiratory units. If Butterworth's case presentations in other specialties are of similar quality I would be tempted to add them to my own bookshelves.DV McG
Heart-Lung Interactions in Health and Disease. Ed S M Scharf, Sharon S Cassidy. (Pp. 1135; \$175, USA and Canada; \$210, all others.) New York: Dekker, 1989. ISBN 08247 7986.

Taking its theme from the Emperor Huang Ti (circa 2600 $\mathrm{BC}$ ), who thought of the heart as "the minister of the monarch, who excels through insight and understanding" and the lungs as "the symbol of the interpretation and conduct of the official jurisdiction," this comprehensive book aims to bring together some of the advances in knowledge of respiration and circulation that have occurred in the last 40 years, and to explore many aspects of their interaction. Over 50 contributors have contributed to 29 sections. Sixteen of these deal with basic physiology, including gas exchange, the pulmonary and bronchial circulations and pulmonary oedema, mechanical function of the cardiorespiratory system, and indirect heart-lung interactions. Five are concerned with pathophysiology and eight with clinical applications, including monitoring and haemodynamic management. Perhaps the viewpoint is slanted a little towards that of the worker in an intensive care unit, who often has to deal with acute dysfunction in heart, lungs, and circulation. Thus there are chapters on "Interaction of respiration and circulation in cardiopulmonary resuscitation" and on "Circulatory effects of positive end-expiratory pressure in patients with acute lung injury." Others may be intrigued by the section dealing with the problems of flight in high performance aircraft and of space travel. There is a valuable and detailed account of methods of non-invasive respiratory monitoring, and their application in ambulant patients and in acute clinical conditions. Each section has its own set of references, though there are relatively few more recent than 1986. The book will be a valuable reference source, and will encourage heart and lung specialists to learn more of each others' territories and of the interface between them. It should be available for consultation in libraries and in specialised units and intensive care areas.-JGR McH 